\title{
Improvement of mental imagery after prism exposure in neglect: a case study
}

\author{
Gilles Rode ${ }^{\mathrm{a}, \mathrm{b}, *}$, Yves Rossetti ${ }^{\mathrm{a}, \mathrm{b}}$, Ling $\mathrm{Li}^{\mathrm{a}}$ and \\ Dominique Boisson ${ }^{\mathrm{a}, \mathrm{b}}$ \\ a Service de Rééducation Neurologique, Hôpital \\ Henry Gabrielle, Hospices Civils de Lyon and \\ Université Claude Bernard, Lyon, France \\ ${ }^{\mathrm{b}}$ Institut National de la Santé Et de la Recherche \\ Médicale, Unité 534: Espace et Action, 16 avenue \\ Lépine, Case 13, 69676 Bron, France
}

\begin{abstract}
Previous work has shown that various symptoms of unilateral neglect, including the pathological shift of the subjective midline to the right, may be improved by a short adaptation period to a prismatic shift of the visual field to the right. We report here the improvement of imagined neglect after prism exposure in a patient with a left unilateral neglect. Despite a strong neglect observed for mental images as well as for conventional tests, the mental evocation of left-sided information from an internal image of the map of France map was fully recovered following prism adaptation to the right. This improvement could not be explained by the alteration of visuomotor responses induced by the prism adaptation. Prism adaptation may therefore act not only on sensory-motor levels but also on a higher cognitive level of mental space representation and/or exploration.
\end{abstract}

Keywords: Unilateral neglect, mental representation, prism adaptation, cognitive neuropsychology

\section{Introduction}

Following right hemisphere damage, patients may show unilateral neglect - a neurological deficit of perception, attention, representation, and/or performing actions within their left-sided space [8]. Most manifestations of this syndrome may be temporarily reduced by vestibular, proprioceptive or tanscutaneous stimulations. Improvement not only concerns extrapersonal and personal neglect, but also anosognosia, somatoparaphrenic delusions and the somatosensory and motor deficits often seen in association with the syndrome (see review in [15]).

${ }^{*}$ Corresponding author: Gilles Rode, Service de Rééducation Neurologique, Hôpital Henry Gabrielle, Hospices Civils de Lyon, Route de Vourles, BP 57, F-69565 Saint-Genis Laval, France. Tel.: +33 04788650 24; Fax: +33 04788650 30; E-mail: gilles.rode@chu-lyon.fr.
Neglect of imagined space may also be temporarily reduced by vestibular stimulation. This improvement may concern imagery-description of familiar squares [6] or geographical maps [10]. In these experiments, the improvement was exhibited as an increased number of items recalled on the previously neglected side with little or no change on the ipsilesional side. This means that the vestibular stimulation does not act through a non-specific mechanism such as an increase in general arousal, neither can it result from a mechanism of global activation of the right hemisphere with a decreasing imbalance between the two hemispheres. Vestibular stimulation in neglect patients may thus act specifically on integrative mechanisms involved in the elaboration of the mental representation of space $[10,11]$.

Guariglia et al. [7] have shown that somatosensory stimulation may also improve imagined neglect. Indeed the effects of transcutaneous electrical neural stimulation on the left side of the neck induced an improvement of performance on the left side in mental representation of objects as well as the free drawing of objects, shape comparison and imagery-description of familiar squares. These results thus suggested that vestibular or somatosensory stimulation may influence the central mechanisms of multimodal integration involved in object as well as in space representation.

Recently, using a short adaptation period to a prismatic shift of the visual field to the right, Rossetti et al. [12] reported an improvement of various symptoms of hemispatial neglect in a group of right brain damaged neglect patients. A reduction of the subjective body midline shift toward the ipsilesional side, shown by these patients, was also noted following the prism adaptation. These findings suggested that, unlike the previous sensory stimulations, the effect of prism adaptation may be conceived as stimulating active processes involved in brain plasticity related to multisensory integration and space representation. However the tasks used in this experiment involved a visuo-manual response (e.g., line cancelling, drawing). Therefore one may ask whether the effect of visuo-motor adaptation could be explained by a di- 
rect alteration of the manual output inherent to the tasks previously explored. An interesting question is whether the improvement observed after prism adaptation would also affect aspects of neglect, which are free from manual responses. We report the improvement of mental imagery after a prism exposure in a neglect patient.

\section{Methods}

\subsection{Subjects}

Three subjects participated in this study. One patient with a left hemispatial neglect and two right-handed control-subjects ( 2 males respectively 56 and 67 years old).

The patient, J.C.G., was a 46 year-old, right-handed man with 14 years of schooling and a long history of hypertension and diabetes. J.C.G. was admitted to hospital on 6 June, 1996 after the sudden onset of a left hemiparesis, with a left homonymous hemianopia and loss of somesthetic sensation on the left side. Head and gaze were deviated to the right, but the patient could move his eyes towards the left both on verbal command and visual pursuit. Speech was mildly dysarthric and there was a right central facial paresis.
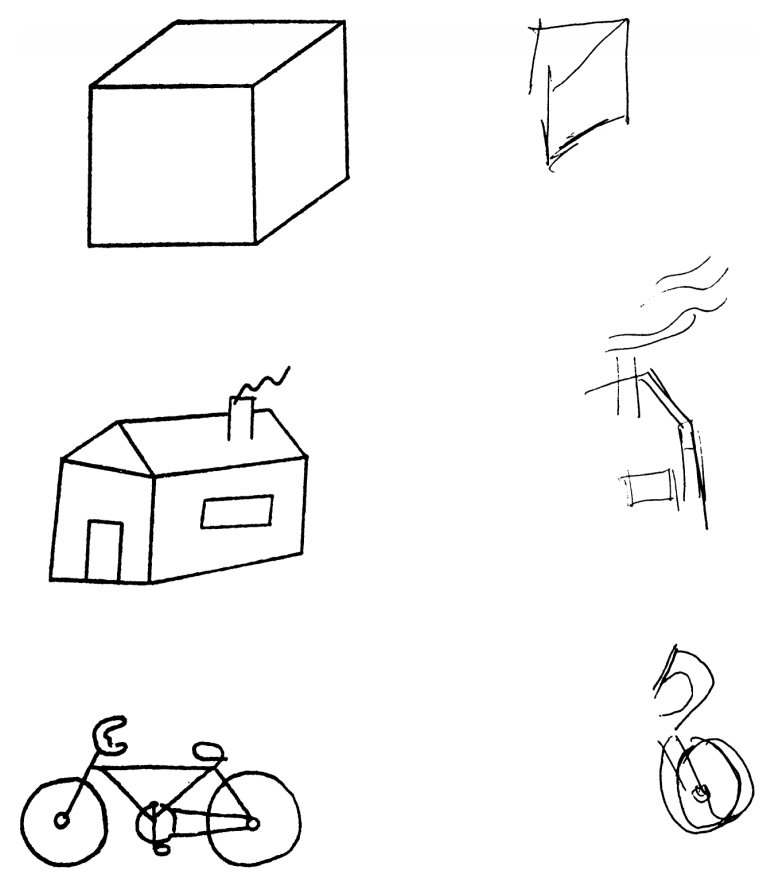

J.C.G.

Fig. 1. Examples of drawings performed by J.C.G. in copying: a cube, a house and a bicycle.
J.C.G. showed a florid left visuo-spatial neglect. For example, the patient omitted most of the left side when asked to draw a daisy or to copy a bicycle or a house (Fig. 1). He failed to complete the clock-face on the same occasion. On a line-cancellation test (1), the patient omitted to cross the left and middle section of the test sheet (28/40). Requested to bisect horizontal lines, he placed his transections significantly to the right of the centre (13). J.C.G. also showed a personal neglect, assessed by clinical examination. A left neglect of imagined space was also noted, when asked the patient to evoke mentally a street or a place of his town. The patient was quite alert, well oriented in time and co-operative. At the time of examination, one month post-onset, the patient was not anosognosic for the motor deficit and the visual deficit had disappeared. Neuropsychological examination also revealed a constructive apraxia dominant for 3D-objects. A CT scan performed 2 months after the stroke revealed a large area of hypodensity in the right hemisphere (Fig. 2). Bilateral carotid and vertebral angiography showed a complete thrombosis of the right internal carotid artery.

\subsection{Procedure}

Three tasks were used to test the effectiveness of prism adaptation in J.C.G. For the control-subjects, only the mental imagery task was performed before and after adaptation.

\subsection{Pointing to straight-ahead}

Subjective body-midline was evaluated by a simple manual pointing task. The patient was seated blindfolded in front of a horizontal box that permitted measurement of the finger movement endpoints with an accuracy of $1 \mathrm{deg}$. He was required to point straightahead while his head was kept aligned with the body's sagittal axis. Ten pointing trials were performed in the pre-test (without goggles) and in the post-test (immediately upon the prism removal), in order to check that prism adaptation had been efficient. The cover of the experimental box allowed the patient to perform free pointing movements either without vision of the arm, or with a partial sight of the arm trajectory (exposure condition).

\subsection{Free drawing of object}

The patient was asked to draw a daisy from memory; The drawing was repeated in three sessions: prior to prism exposure (Pre-test), on prism removal (Post-test) and 24 hours later (Late-test). 


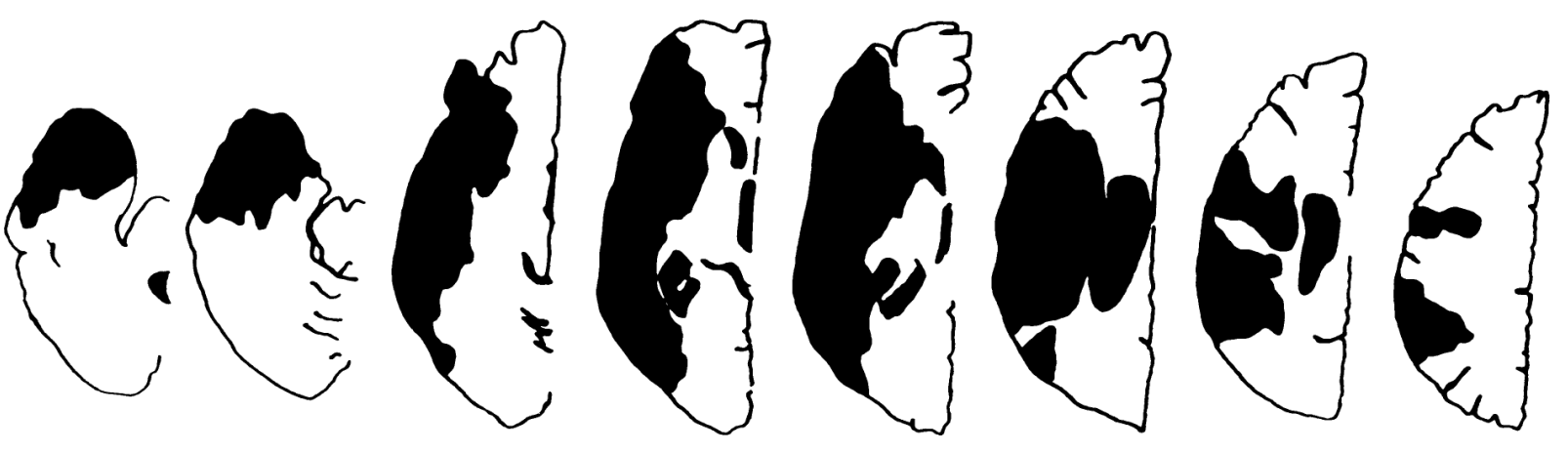

Fig. 2. Extent of the right-hemisphere lesion plotted on axial templates obtained from a CT-scan performed 1 month after onset. Section shows a large lesion in the right hemisphere, centred around the temporo-parieto-occipital carrefour, respecting the thalamic region and encroaching upon the external capsule and the putamen.

\subsection{Mental imagery task}

Subjects were asked to mentally evoke the map of France and to name as many towns as possible on the map that they could 'see' within 2 minutes (see details in [8]). The patient was tested before adaptation (Pre test), immediately after (Post test) and 24 hours after (Late test) prism exposition.

Performance was rated by a global score corresponding to the total number of towns named within 2 minutes (total score). In addition, 2 scores were computed according to the geographical position of each named town, either on the right of the map central axis (Perpignan-Lille)(right score), or on the left of this axis (left score). Four sub-scores relation to the geographical position of named towns on each lateral quarter of the map were also compared to test for extreme West and extreme East effects.

Results were subjected to three different analyses: (i) an ANOVA comparison of the mean horizontal distance of named towns from the Perpignan-Lille axis. In this analysis, the horizontal deviation was measured in mm on a standard map of France (scale: 1/5000000; $1 \mathrm{~cm}=50 \mathrm{~km}$ ), (ii) a comparison of the right and left scores with a Chi-2 analysis, and (iii) a comparison of each sub-score for each side with a Chi-2 analysis.

\subsection{Prism adaptation}

Subjects were exposed to a shift of the visual field to the right produced by prismatic lenses. The goggles were fitted with wide-field, wedge lenses creating an optical shift of $10 \mathrm{deg}$. The exposure period consisted in 50 pointing responses to visual targets presented $10 \mathrm{deg}$ to the right or to the left of the objective body midline. During the prism exposure, subjects were asked to point at a fast but comfortable speed; they could see the target, the second half of their pointing trajectory and their terminal error. Their head was kept aligned with the body's sagittal axis by a chin-rest and controlled by an investigator. The total duration of this exposure was about 3 minutes.

This experimental procedure was applied one month post-onset.

\section{Results}

\subsection{Pointing to straight-ahead}

In the Pre-test, the patient's straight-ahead was initially shifted to the left (mean: $-5^{\circ}$; Standard Error of the Mean (SEM): 1.26). In the Post-test, the patient demonstrated a larger straight-ahead shift to the left (mean: $-14^{\circ}$; SEM: 0.52). Comparison by $\mathrm{t}-$ test of mean deviation observed before and following the adaptation revealed a very significant difference $(t(9)=7.73 ; p<0.0001)$ indicating that J.C.G. could adapt to a lateral shift of the visual field to the right.

\subsection{Free drawing of object}

In the Pre-test, the patient drew a daisy restricted to a right half. In the Post-test, the patient draw a entire daisy with a surprising larger left half than the right and in the Late-test, a incomplete daisy with a smaller left half than the right (Fig. 3).

\subsection{Mental imagery task}

For the 2 control-subjects, the mean total score of named towns given prior to prism exposure (Pre-test) was 47.5 (respectively 22.5 for the right and 25 for the left). In the Post-test, the value was 44.5 (respectively 


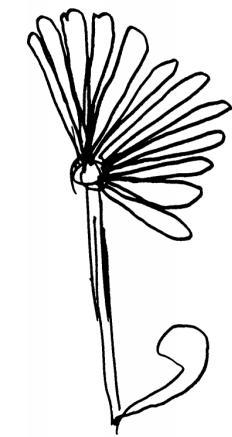

PRE

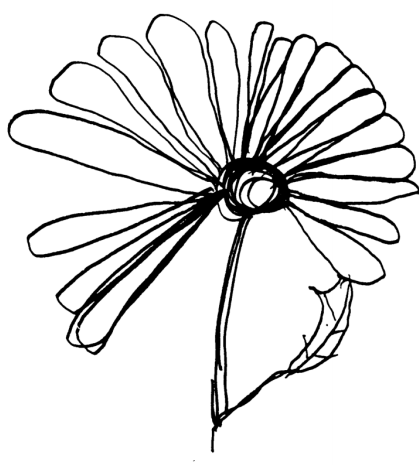

Post

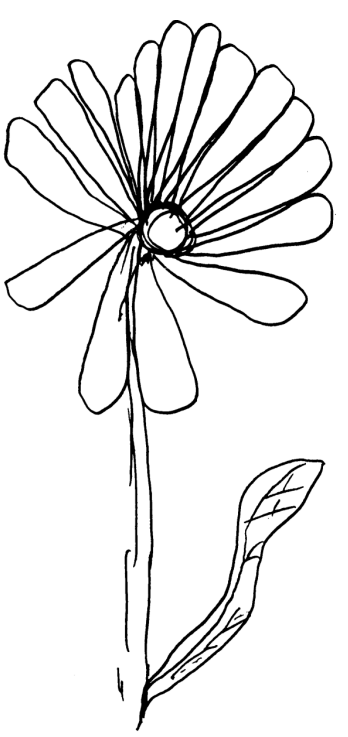

LATE J.c.G.

Fig. 3. Drawings of a daisy, from memory, in three conditions: prior to prism exposure (Pre-test), immediately after removing the goggles (Post-test), and after a delay of about 24 hours following the prism exposure (Late-test).

23 for the right and 21.5 for the left). Morever the comparison of the difference between the right and left scores showed no difference prior to prism exposure (-2.5) and after (1.5). For the 2 control-subjects, the mental evocation of the map of France thus was not modified by the prism adaptation.

For J.C.G., scores obtained before (Pre-test) and after prism adaptation (Post-test and Late-test) are indicated in Table 1. The total score of named towns given prior to prism exposure (Pre-test) was increased in the Post-test (on prism removal) and in the Late-test (after 24 hours). The geographical locus of responses also differed across conditions: in the Pre-test, most towns evoked by J.C.G. were located on the right of the midline axis of the map of France (Perpignan-Lille axis). The patient evoked only 3 towns on the left and 2 on the vertical axis. In the Post-test, J.C.G. evoked the same number of towns on the right of the Perpignan-Lille axis (which differed, however, in location from those previously named in the Pre-test), but he evoked 21 towns on the left of the Perpignan-Lille axis and 3 on the vertical axis. When tested 24 hours after prism exposure (Late-test), J.C.G. again named far fewer towns on the left side and the evoked towns were mostly located on the right of of the PerpignanLille axis (Fig. 4).

A Chi-2 test performed on the left-right scores obtained in the 3 sessions showed a significant effect $\left(\chi^{2}(2)=15.63, p<0 ; 0005\right)$. Additional test performed between each pair of sessions showed a sig-
Table 1

Scores of towns evoked by J.C.G. from mental evocation of the map of France, within 2 minutes, prior to prism exposure (Pre-test), immediately after prism adaptation (Post-test) and after a delay about 24 hours following the prism exposure (Late-test). The vertical line $\mathrm{P}-\mathrm{L}$ indicates the Perpignan-Lille axis.

\begin{tabular}{llclcc}
\hline & \multicolumn{2}{c}{ Left scores } & \multicolumn{2}{c}{ Right scores } & Total score \\
\hline Pre-test & 0 & 3 & 12 & 6 & 21 \\
Post-test & 9 & 13 & 15 & 2 & 39 \\
Late-test & 0 & 6 & 14 & 14 & 34 \\
\hline
\end{tabular}

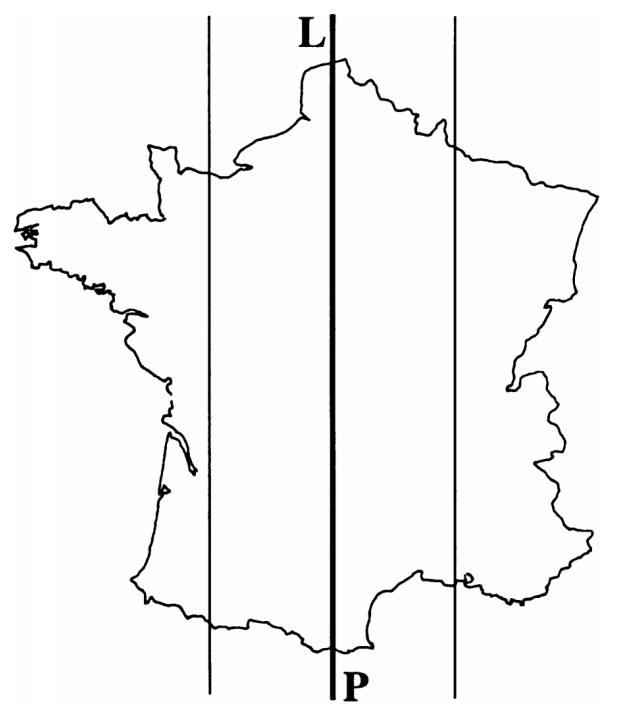

nificant difference between the Pre- and the Post-tests $\left(\chi^{2}(1)=9.41, p<0.005\right)$ and between the Post- 


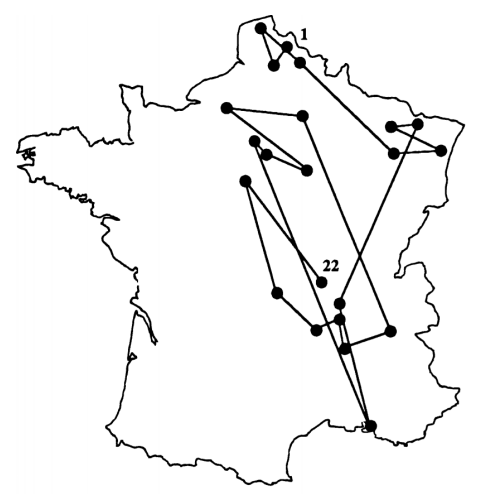

Pre

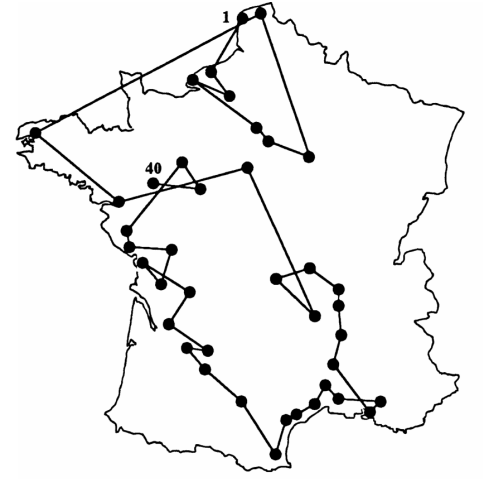

Post

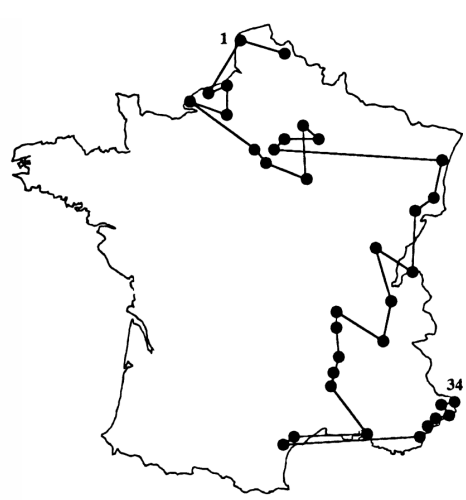

LATE

Fig. 4. Maps of France described from mental evocation in three conditions: prior to a prism exposure (Pre-test), immediately after removing the goggles (Post-test) and after a delay of about 24 hours following the prism exposure (Late-test). The filled circles indicate the geographical loci of the different responses and the bold number indicates the total number of responses.

and the Late-tests $\left(\chi^{2}=10.83, p<0.001\right)$. The test performed between the Pre- and the Late-tests confirmed that there was no difference between these two sessions $\left(\chi^{2}=0.11, p>0.50\right)$.

In addition, comparisons were performed on the subscores obtained for each quarter of the map. The global comparison between the three sessions showed a significant effect $\left(\chi^{2}(6)=27.12, p<0.0001\right)$. The additional comparison performed between the Preand the Post-tests was significant $\left(\chi^{2}(3)=13.19\right.$, $p<0.005)$. Again, the comparison between the Postand the Late-tests was significant $\left(\chi^{2}(3)=20.57\right.$, $p<0.0001)$ and the comparison between the Preand the Late-tests revealed no significant difference $\left(\chi^{2}(2)=1.36, p>0.50\right)$. Interestingly, the highest contributions to the $\chi^{2}$ in the global comparison of four subscores were found for the leftmost quarter (3.75) and for the right-most quarter $(-3.52)$ of the Post-test (see Fig. 4).

Computation of the mean horizontal deviation from the map central axis also provided variance estimates, which were used to assess the horizontal extent of attention across the mental map. The square root of these variances are displayed in Fig. 5 as SD whiskers. The pair-comparison of these variances revealed that the Pre-test variance significantly differed from the two others $(F(40,22)=1.80, p<0.05$ and $F(34,22)=$ $1.75, p<0.01)$. The Post- and Late-tests variances did not differ significantly $(F(40,34)=1.03, p>0.25)$. This suggested that the spatial span of attention was increased after adaptation and that this increase was maintained over 24 hours. In addition, Fig. 5 shows that the evolution of the mean position of towns named and of its variance can be dissociated: prism adaptation produced a more lasting effect on the horizontal width explored on the map than on the mean distance to midline.

\section{Discussion}

Before prism exposure, J.C.G. showed a left visual hemineglect on conventional tasks and a left neglect of imagined space on a task requiring him to report from an imagined representation of the map of France without an ipsilesional shift of the subjective midline. In J.C.G., the same side of space (left) was affected by representational and visuospatial neglect. But the shift of the subjective midline was in the opposite direction, further supporting that this particular manifestation can be dissociated from other symptoms of neglect [5].

The neglect of imaginal space shown by J.C.G. in the mental evocation of the map of France is similar to that reported previously in 14 neglect patients [10, 11]. It suggests a distorted representation of the geographic map, which could be conceived of as a topologically structured representation split between the two cerebral hemispheres [2, 3].

Immediately after prism adaptation, modifications of the mental evocation were evidenced: (i) the total number of towns named by the patient within 2 minutes was increased, suggesting that the prism adaptation facilitated the mental evocation of the map. (ii) This increase concerned mainly the evocation of towns located on the left half of map, suggesting a regression of 


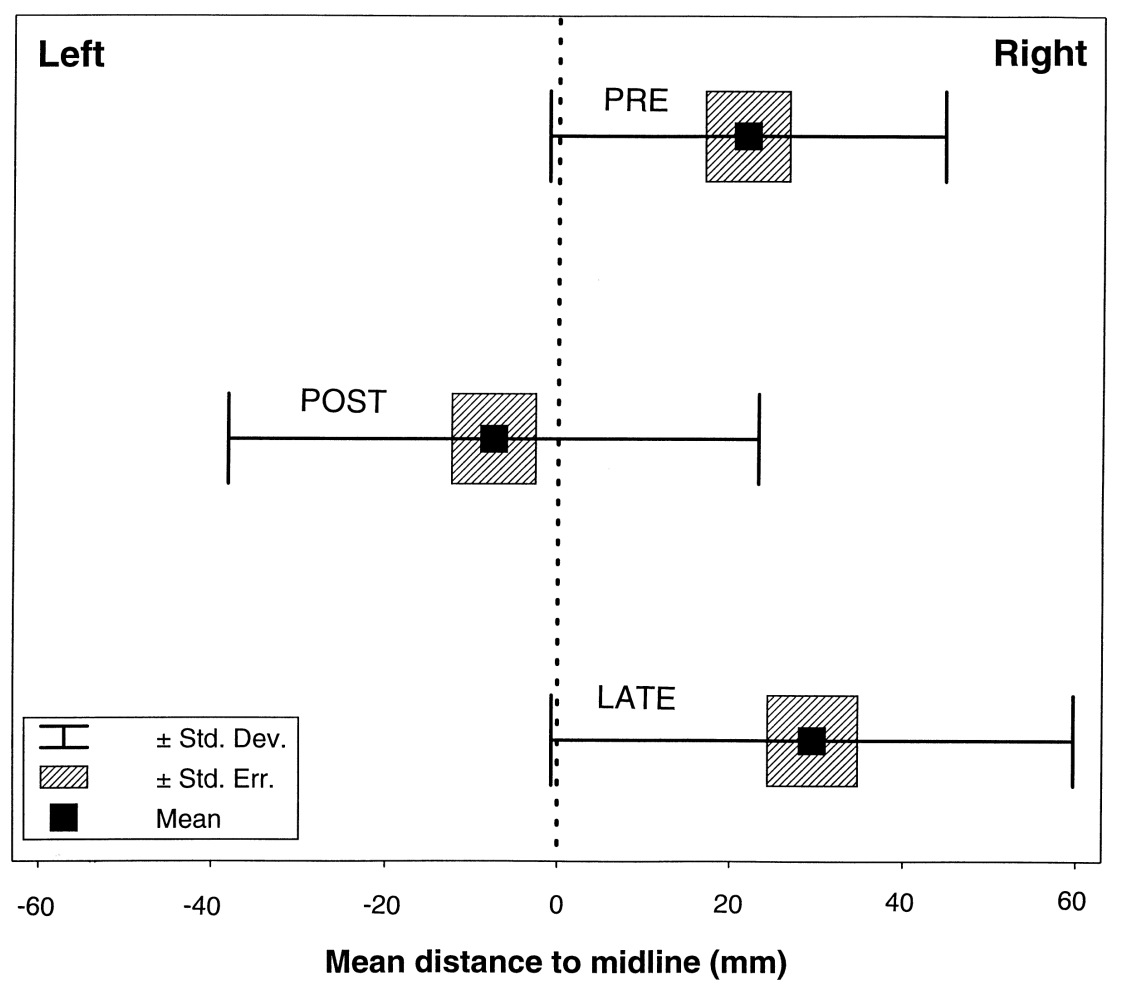

Fig. 5. Mean horizontal distance of named towns measured from the map midline (Perpignan-Lille axis) prior to prism exposure (Pre-test), immediately after prism adaptation (Post-test) and after a delay about 24 hours following the prism exposure (Late-test). Box indicates standard errors of the mean and the whiskers indicates standard deviations. Both the mean deviation to midline and its variance were affected by the adaptation in the Post-test. The effect of adaptation lasted over $24 \mathrm{~h}$ only for the horizontal variance.

left representational neglect and (iii) no town is evoked on the east-most part of map, suggesting a significant shift of mental evocation toward the left side of the image and a paradoxical 'neglect' of the right side. Morever following the adaptation, the patient showed improved drawing with reduced asymmetry of the daisy. This improvement was also paralleled by the significant increase of the straight-ahead shift toward the left side, which again suggests that these two symptoms can be dissociated. The reversal effect observed after prism adaptation strongly supports the idea that the effect of this manipulation on neglect is attributable to a specific directional mechanism, and not just to a general improvement of cognitive functions.

After a delay of about 24 hours, following the prism exposure, the previous modifications of mental evocation had disappeared and the patient again showed left neglect of imaginal space, comparable with performance prior to adaptation. However the total number of towns named remained larger than before prism exposure, probably reflecting learning of the mental task. On the other hand, the improvement in daisy drawing was partially maintained. The difference between drawing from memory and mental evocation may be explained by the involvement of a manual response in daisy drawing.

In J.C.G., a short adaptation period to a prismatic shift of the visual field to the right significantly improved the mental evocation of left-sided information from a geographical space, and the free drawing from memory. We have already reported an improvement in a task of drawing from memory or in copying following prism exposure in a group of 6 right brain damaged neglect patients [12]. In this case, a novel effect of prism adaptation is reported in a task of mental imagery. In this task, the subject had to generate an inner image of map from the long-term memory and explore it. It should be noticed that this level of space representation clearly differs from the sensory-motor level, directly stimulated by prism adaptation. The reduction of imaginal neglect shown by J.C.G. after the prism adaptation suggests that the stimulation of active processes involved in the plasticity of sensori-motor correspondences by the prism adaptation can also influence cognitive processes at the level of mental representation. This result thus further supports the idea 
[12] that the process of prism adaptation could activate brain functions related to multisensory integration and higher spatial representations.

It should be remembered that the generalisation of prism adaptation has been shown to be rather restricted in normals. In conditions comparable to the ones used here (i.e., with head movements prevented by a chin rest and a short prism exposure), there was usually no generalisation of the adaptation to the arm that was not used during the exposure. In addition, short-term prism exposure, as used in our experiment, does not usually give rise to a change in the resting gaze posture. These data strongly contrast with the high degree of generalisation observed in patients with unilateral neglect. Two hypotheses may account for these results. The first hypothesis is that the general sensitivity to the prismatic shift would be higher in patients than in controls. Accordingly, the amount of after-effect found in patients is twice that in normals [12]. In addition, it is usual that patients exhibit more variable performance than normals, which suggests that their spatial functions are more labile than normals. For these two reasons a deeper effect of adaptation should be expected in patients, that may generalise to non-motor tasks. The second hypothesis is derived from the observation that patients do not adapt to a leftward visual shift [12]. This effect would indicate that neglect patients have a tendency to react to prism in such a way as to alter their sensori-motor system only in a beneficial way. This tendency may rely on the action of error signals generated by the neglect syndrome on the spatial representation structures. Error signals linked to neglect that are provided to the brain during the prism exposure, may have a synergistic effect with the adaptation process proper and potentialise the effect of the prisms. Morever a related issue concerns the lack of a prism adaptation effect on the control subjects.

In addition, this case raises an intriguing question regarding the effect of prism adaptation on mental imagery, and the format of mental representation (4). In J.C.G., does prism exposure facilate the elaboration of a symmetrical image of the map (3), or the mental exploration of an entire map representation (14)? The mental shift shown by the patient following prism adaptation and the poorer exploration of the right part of the map may suggest that the adaptation facilitated the mental exploration rather than the mental generation of the left part of the image. The effects of adaptation may thus involve a 'mental shift' of selective attentional processes to the neglected-side of an inner image.
Lastly, the variance of the horizontal position of the towns named in the three tests suggested that prism adaptation induced an increase of the horizontal span of attention applied to mental images. Three common features can be found between the present result and the study of actual oculomotor exploration of space [9]. First, the total spatial area of exploration was significantly enlarged in both studies. Second, the bias to one side of the median axis was reduced after prism exposure (the present study) as well as after neck vibration or vestibular stimulation [9]. Third, the horizontal span of spatial exploration was increased for both mental (this study) and oculomotor [9] tasks. In addition, the prism adaptation resulted not only in an increase in the number of towns named on the left but also in a significant decrease in the number of towns reported on the right-most part of the map.

\section{Acknowledgements}

This work was supported by the Région RhôneAlpes (Thématiques Prioritaires), by the Hospices Civils de Lyon (P.H.R.C. ${ }^{\circ}$ 95.035) and by Inserm (PROGRES). We thank R. MacIntosh for his comments on the manuscript.

\section{References}

[1] M.L. Albert, A simple test of visual neglect, Neurology 23 (1973), 658-664.

[2] E. Bisiach and C. Luzzatti, Unilateral neglect of representational space, Cortex 14 (1978), 129-133.

[3] E. Bisiach and A. Berti, The directional coding of reaching movements. A visuomotor conception of spatial neglect, in: Neurophysiological and Neuropsychological Aspects of Spatial Neglect, M. Jeannerod, ed., North Holland, Amsterdam, 1987, pp. 183-201.

[4] M.J. Farah, K.M. Hammond, D.N. Levine and R.M. Calvino, Visual and spatial imagery: dissociable systems of representations, Cognitive Psychology 20 (1988), 439-462.

[5] A. Farné, F. Ponti and E. Ladavas, In search of biased egocentric reference frames in neglect, Neuropsychologia 36 (1998), 611-623.

[6] G. Geminiani and G. Bottini, Mental representation and temporary recovery from unilateral neglect after vestibular stimulation, J. Neurol. Neurosurg. Psychiat. 55 (1992), 332-333.

[7] C. Guariglia, G. Lippolis and L. Pizzamiglio, Somatosensory stimulation improves imagery disorders in neglect, Cortex 34 (1998), 233-241.

[8] K.M. Heilman, R.T. Watson and E. Valenstein, in: Clinical Neuropsychology, K.M. Heilman and E. Valenstein, eds, Oxford University Press, New York, 1985, pp. 243-293.

[9] H.-O. Karnath, M. Fetter and J. Dichgans, Ocular exploration of space as a function of neck proprioceptive and vestibular input-observations in normal subjects and patients with spa- 
tial neglect after parietal lesions, Exp. Brain Res. 109 (1996), 333-342.

[10] G. Rode and M.T. Perenin, Temporary remission of representational hemineglect through vestibular stimulation, $\mathrm{Neu}$ roreport 5 (1994), 869-872.

[11] G. Rode, M.T. Perenin and D. Boisson, Effets de la stimulation vestibulaire sur la négligence de l'espace représenté, in: Le Cortex Vestibulaire, M. Collard, M. Jeannerod and Y. Christen, eds, Irvinn, Paris, 1996, pp. 109-116.

[12] Y. Rossetti, G. Rode, L. Pisella, A. Farné, L. Li, D. Boisson and M.T. Perenin, Prism adaptation to a rightward optical deviation rehabilitates left hemispatial neglect, Nature $\mathbf{3 9 5}$ (1998), 166-169.

[13] T. Schenkenberg, D.C. Bradford and E.T. Ajax, Line bisection with neurologic impairement, Neurology 30 (1980), 509-517.

[14] L. Trojano and D. A. Grossi, Critical review of mental imagery defects, Brain and Cognition 24 (1994), 213-243.

[15] G. Vallar, C.Guariglia and M.L. Rusconi, Modulation of the neglect syndrome by sensory stimulation, in: Parietal lobe contribution to orientation in 3D space, P.Thier and H.O. Karnath, eds, Springer Verlag, Heidelberg, 1997, pp. 555579. 


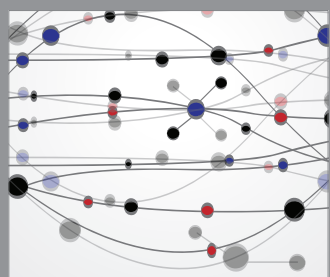

The Scientific World Journal
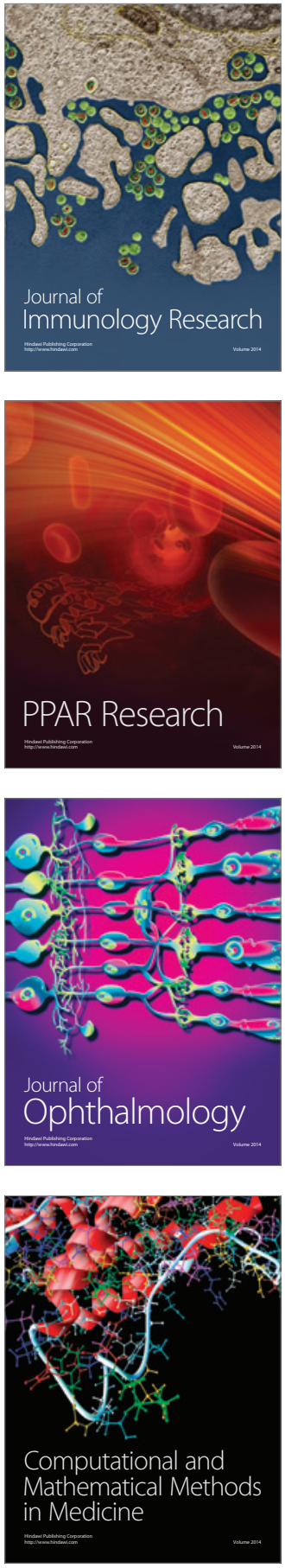

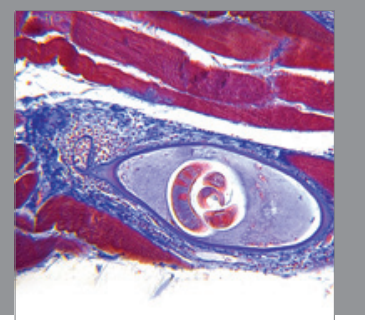

Gastroenterology

Research and Practice
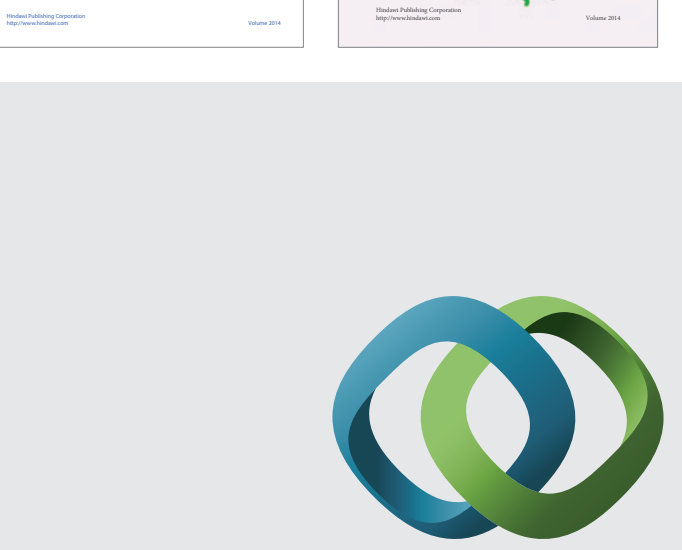

\section{Hindawi}

Submit your manuscripts at

http://www.hindawi.com
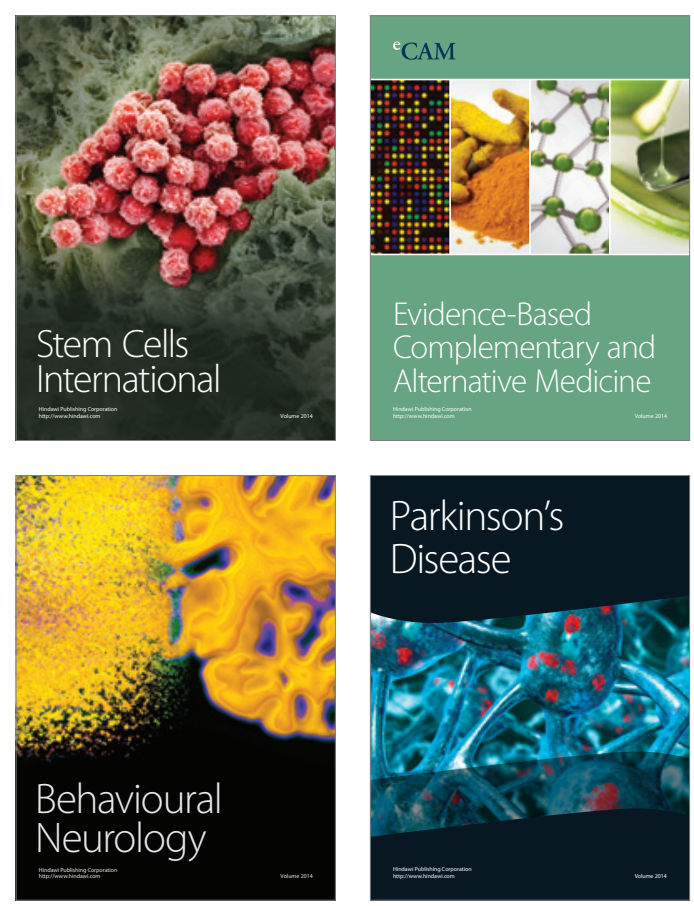

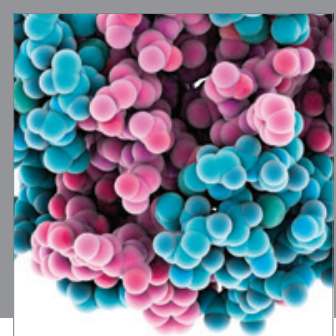

Journal of
Diabetes Research

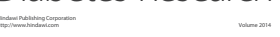

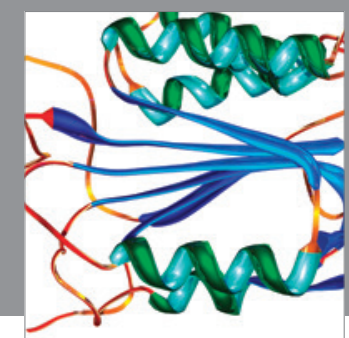

Disease Markers
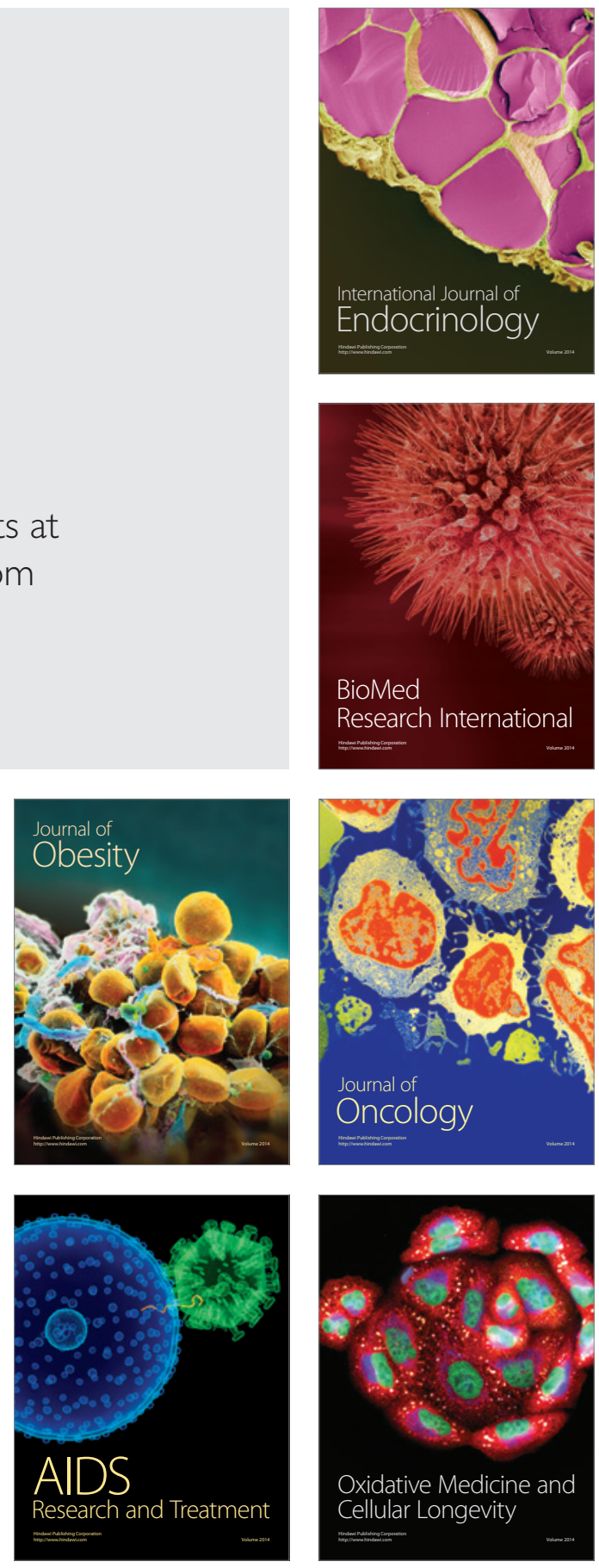